\title{
Simultaneous Suppression of IMD3 and IMD5 in Space TWT by IMD3 and 2HD Signal Injection
}

\author{
Dongming Zhao, ${ }^{1}$ Huijuan Liu, ${ }^{2}$ Kewen Xia, ${ }^{1}$ Shi Li, ${ }^{3}$ and Xiaoxu Shi ${ }^{1}$ \\ ${ }^{1}$ School of Information Engineering, Hebei University of Technology, Tianjin 300401, China \\ ${ }^{2}$ Tianjin Broadcasting TV and Film Institute, Tianjin 300112, China \\ ${ }^{3}$ Institute of Electronics, Chinese Academy of Sciences, Beijing 100080, China \\ Correspondence should be addressed to Kewen Xia; kwxia@hebut.edu.cn
}

Received 13 November 2016; Accepted 12 December 2016; Published 10 January 2017

Academic Editor: Wen-Ming Zhang

Copyright (C) 2017 Dongming Zhao et al. This is an open access article distributed under the Creative Commons Attribution License, which permits unrestricted use, distribution, and reproduction in any medium, provided the original work is properly cited.

\begin{abstract}
This paper presents a signal injection technology showing significant reductions in both 3rd-order and 5th-order intermodulation distortions (IMD3 and IMD5) in space traveling wave tube (STWT). By applying the IMD3 to the IMD5 ratio (TFR) as measures of location, the simultaneous suppressions of IMD3 and IMD5 in TWT are achieved by second harmonic distortion (2HD) and IMD3 injection. According to the research on theoretical analysis and computer simulation, the optimum amplitude and phase parameters of the injected signal for maximum simultaneous suppressions are obtained. Then an experiment system is established based on vector network analyzer, optimum TFR are $2.1 \mathrm{~dB}$ and $12.5 \mathrm{~dB}$, respectively, by second harmonic and IM3 injection, and the output powers of IMD3 and IMD5 were decreased. TFR with IMD3 injection is smaller than that with second harmonic injection in STWT, and the experiment system is more straightforward and easy to operate. Thus, the IMD3 injection performs better than that of second harmonic injection to suppress IMD5s for the narrow-band STWT.
\end{abstract}

\section{Introduction}

Traveling wave tube (TWT) amplifier is widely used in the fields of communication, signal processing, radar, electronic warfare, and so forth. Space TWT is the core component of satellite communication system, and it plays a crucial role in power amplification in the field of space technology, high gain, and excellent linearity is an essential prerequisite for its normal operation. Unfortunately, TWT exhibits nonlinear characteristic and therefore generates unwanted intermodulation distortion (IMD) and second harmonic distortion (2HD). Among various distortions, harmonics can be eliminated by the filter, but the 3rd-order (IMD3) and 5th-order intermodulation (IMD5) fall on the main signal band and cannot be easily filtered out. Meanwhile, the IMD signal severely limits the fundamental energy of TWT and reduces the output power; thus it causes lower overall system performance. Earlier articles show that the mechanism of distortion suppression by signal injection is the destructive interference of the injected signal with nonlinearly generated distortion product [1-4]. Although the experimental condition of signal injection technology is relatively complex, it has very noticeable effect for distortion suppression in several experimental environments, so signal injection technology is used to study simultaneous suppressions in both IMD3 and IMD5 of STWT in this paper.

The MUSE theoretical model [1] was presented by Wöhlbier et al. The models can clearly describe the waveforms regarding injected and nonlinearly generated components in TWT. The comparison of reduction with secondorder versus third-order signal before distortion method for TWT is studied by Singh in detail in her experiment [5-7]. In other areas of electronic device, An optoelectrical predistortion optical transmitter system is designed by Lee et al. [8]. This presents that the simultaneous suppressions of IMD3 and IMD5 are achieved by choosing an appropriate bias current and RF input power for the LD. The feedback second harmonic injection methods for simultaneous reduction of 
IMD3 and IMD5 in bisection laser diode have been studied [9], and a novel distortion reduction scheme for gain lever laser diodes has been presented $[10,11]$.

In recent years, there are some new research results about signal injection including the improvement of the fundamental signal power of TWT by harmonic injection [4, 12 ], the harmonic and intermodulation distortions associated with two-tone modulation [13], the suppression of IMD3 by harmonic injection [14], and the particle swarm optimization algorithm (PSO) which are applied to suppress IMD $[15,16]$. Furthermore, the previous research results of our research group show that IMD3 and 2HD injection approaches are all available in suppressing IMD3 components. IMD3 injection performs better than $2 \mathrm{HD}$ injection for narrow-band space TWT $[17,18]$. The research on simultaneous reductions in both IMD3 and IMD5 of STWT by signal injection has not been conducted, which will be concerned and investigated in this paper.

First of all, many abbreviations in this paper are explained. FUN is the abbreviation of fundamental signal, and FUN- and FUN+ represent lower and upper fundamental, respectively, in two-tone signal. IMD3- and IMD3+ are lower and upper third-order intermodulation distortion components, and IMD5- and IMD5+ are lower and upper fifth-order intermodulation distortion components. Initial power and initial fundamental frequency represent input power and frequency of two-tone fundamental signal, they are variable, and the initial power and frequency variation of fundamental signal can influence output power of FUN, IMD3, and IMD5 under optimum injection and without signal injection.

\section{Theoretical Analysis}

We use the proposed nonlinear model on analog amplifier to analyze the suppression in IMD3 and IMD5 theoretically. The amplifier nonlinearity can be expressed with a Taylor series equation $[8,9,19]$ connecting the output power $P$ to input signal $I_{\text {in }}$ by (1); $g_{m 1} g_{m 2} g_{m 3}$ are constants.

$$
P=g_{m 1} I_{\text {in }}+g_{m 2} I_{\text {in }}{ }^{2}+g_{m 3} I_{\text {in }}{ }^{3} .
$$

The input signal (two-tone fundamental signal) consists of two tones given by expression $I_{\text {in }}=A_{1} \cos \omega_{1} t+$ $A_{2} \cos \omega_{2} t$, the amplitude and phase are $A_{1} A_{2}$ and $\omega_{1} \omega_{2}$, and the amplitude represents the power of signal in terms of power amplifier. After being amplified, the IMD3 component at $2 \omega_{2}-\omega_{1}$ is $0.75 A_{1} A_{2}^{2} g_{m 3} \cos \left(2 \omega_{2}-\omega_{1}\right) t$, and IMD5 component at $3 \omega_{2}-2 \omega_{1}$ is

$$
\begin{aligned}
& 0.5 g_{m 4} A_{2}^{3} A_{11} \cos \left[\left(3 \omega_{2}-2 \omega_{1}\right) t+\phi_{1}\right] \\
& +0.625 A_{1}^{2} A_{2}^{3} g_{m 5} \cos \left(3 \omega_{2}-2 \omega_{1}\right) t .
\end{aligned}
$$

A IM3 signal of amplitude $A_{21}$ and phase $\phi_{21}$ at $2 \omega_{2}-$ $\omega_{1}$ is injected into the amplifier together with the two-tone fundamental signal; the input signal is

$$
\begin{aligned}
I_{\text {in }}= & A_{1} \cos \omega_{1} t+A_{2} \cos \omega_{2} t \\
& +A_{21} \cos \left[\left(2 \omega_{2}-\omega_{1}\right) t+\phi_{21}\right] .
\end{aligned}
$$

The IMD3 output power $\left(2 \omega_{2}-\omega_{1}\right)$ at the output of amplifier is expressed as

$$
\begin{aligned}
& 0.75 A_{2}^{2} A_{1} g_{m 3} \cos \left(2 \omega_{2}-\omega_{1}\right) t+\left[A_{21} g_{m 1}\right. \\
& +1.5 A_{21} g_{m 3} A_{1}^{2}+1.5 A_{21} g_{m 3} A_{2}^{2} \\
& \left.+0.75 A_{21} g_{m 3} A_{21}^{2}\right] \cos \left(2 \omega_{2} t-\omega_{1} t+\phi_{21}\right) .
\end{aligned}
$$

The IMD5 output power $\left(3 \omega_{2}-2 \omega_{1}\right)$ at the output of amplifier is written as

$$
\begin{aligned}
0.625 g_{m 5} A_{1}{ }^{2} A_{2}{ }^{3} \cos \left(3 \omega_{2}-2 \omega_{1}\right) \\
+\left[3.75 g_{m 5} A_{1}{ }^{2} A_{2}{ }^{3} A_{21}{ }^{2}+1.25 g_{m 5} A_{2} A_{21}{ }^{4}\right. \\
+3.75 g_{m 5} A_{1}^{3} A_{2} A_{21} t+3.75 g_{m 5} A_{1} A_{2}{ }^{3} A_{21} \\
\left.+1.5 g_{m 3} A_{1} A_{2} A_{21}\right] \cos \left(3 \omega_{2} t-2 \omega_{1} t+2 \phi_{21}\right) \\
+\left[3.75 g_{m 5} A_{1} A_{2} A_{21}{ }^{3}+1.25 g_{m 5} A_{2} A_{21}{ }^{4}\right. \\
+3.75 g_{m 5} A_{1}{ }^{3} A_{2} A_{21}+3.75 g_{m 5} A_{1} A_{2}{ }^{3} A_{21} \\
\left.+1.5 g_{m 3} A_{1} A_{2} A_{21}\right] \cos \left(3 \omega_{2} t-2 \omega_{1} t+\phi_{21}\right) .
\end{aligned}
$$

All parameters in (4) and (5) are known. The output power of IMD3 $2 \omega_{2}-\omega_{1}$ can be suppressed to zero by appropriately adjusting $A_{21}$ and $\phi_{21}$, but they are not the optimal parameters to completely suppress IMD5 $3 \omega_{2}-2 \omega_{1}$ at the same condition.

It can be seen from (5) that the expression of IMD5 is quite complicated. If we appropriately adjust the parameters theoretically $3 \omega_{2}-2 \omega_{1}$ can be suppressed to zero by observing expression. Maximum suppressions in IMD3 and IMD5 $\left(2 \omega_{2}-\omega_{1}\right.$ and $\left.3 \omega_{2}-2 \omega_{1}\right)$ do not occur simultaneously by a set of amplitude and phase of injected signal, so we need to find the optimal amplitude and phase parameter to make compromises between them. By introducing the definition of IMD3 to the IMD5 ratio (TFR), and choosing the optimal operating point of the injected amplitude and phase where the TFR has minimum, the optimum signal injection conditions for simultaneous suppressions in the IMD3 and IMD5 can be determined. TFR $(\mathrm{dB})$ is indicated to measure the relative power of IMD3 and IMD5 and represents multiple relationships between the output power of IMD3 and IMD5; it is the logarithmic representation of output power ratio.

$L_{\text {out,IM3 }}$ and $L_{\text {out,IM5 }}$ represent the output power of IMD3 and IMD5, respectively; the unit is $\mathrm{dBm} . W_{\mathrm{IM} 3} / W_{\mathrm{IM} 5}$ represent IMD3 and IMD5 output power ratio.

$$
\begin{aligned}
\mathrm{TFR}_{\text {IM3 to IM5 }} & =L_{\text {out,IM3 }}-L_{\text {out }, \text { IM5 }}[\mathrm{dB}] \\
& =10 \lg W_{\text {IM3 }}-10 \lg W_{\text {IM5 }} \\
& =10 \lg \left(W_{\text {IM3 }} / W_{\text {IM5 }}\right) .
\end{aligned}
$$

When the optimal operating point of the injected amplitude and phase is found where TFR $\mathrm{IM}_{3}$ to IM5 has minimum, the relative power of IMD5 and IMD3 and the difference of output power are minimum. The simultaneous suppression effect in the IMD3 and IMD5 is observed. 


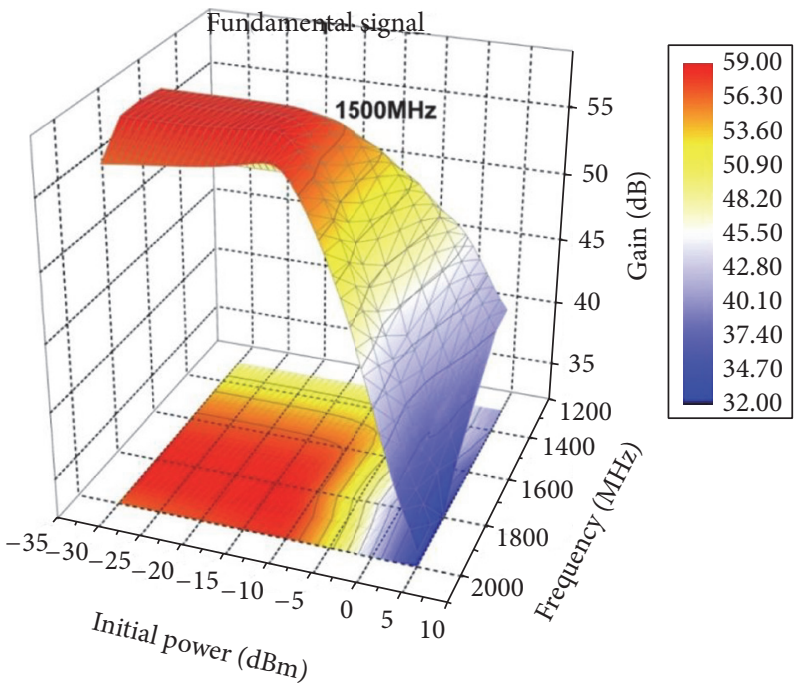

(a)

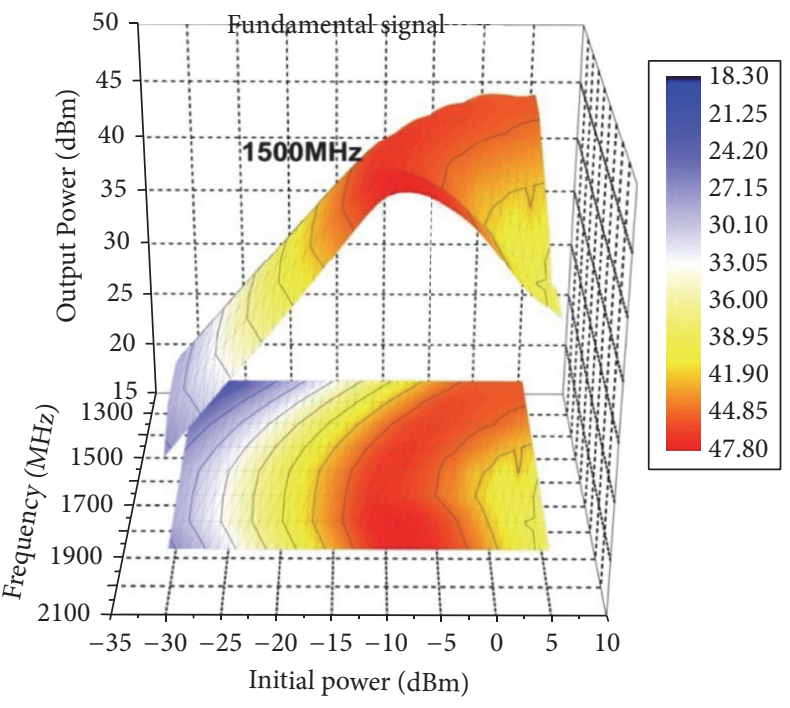

(b)

FIGURE 1: (a) 3D plot of output gain; (b) 3D plot of output power according to the fundamental frequency and initial power.

\section{Simulation Results and Discussions}

Serious nonlinear distortion components appear at the output port after STWT begin to work on saturation state. The third-order intermodulation frequencies $2 \omega_{1}-\omega_{2}$ and $2 \omega_{2}-\omega_{1}$ lie closest to the fundamental frequency among all intermodulation frequencies $m \omega_{1}+n \omega_{2}$, Thus IMD3 are the main contributors to nonlinear distortion and have the most significant effect on the output power of the fundamental signal. IMD5 $\left(3 \omega_{1}-2 \omega_{2}\right.$ and $\left.3 \omega_{2}-2 \omega_{1}\right)$ are also in the working frequency band of STWT, the effect on fundamental signal is next to IMD3, and both of them are the main performance of nonlinear distortion in STWT.

The study on signal injection to simultaneously suppress the IMD3 and IMD5 has been held all along to improve linearity performance of STWT significantly. Wöhlbier et al. study comprehensively physical principles of signal injection technique for TWT [1] and propose TWT nonlinear spectral model LATTE and S-MUSE. According to the nonlinear spectral model, various simulation experiments on the suppression of IMD3 and IMD5 are done in this paper under several types of signal injection. By adjusting the signal power, phase, and frequency precisely, the performance and change situation of output parameters such as power and frequency of STWT are shown.

3.1. Fundamental Signal Parameters. The 3D plots of the output gain and power of fundamental signal according to the fundamental frequency and initial power are shown in Figure 1. When the fundamental frequency is relatively low (less than $1.5 \mathrm{GHz}$ ), the gain effect is not evident. After the frequency increases to $2.0 \mathrm{GHz}$, and initial power is greater than $-5 \mathrm{dBm}$, the gain will quickly be reduced to $30 \mathrm{~dB}$. However, After the frequency is selected as $1.5 \mathrm{GHz}$, the amplification performance gets better, and output power of STWT is more than $30 \mathrm{dBm}$. Under smaller initial power

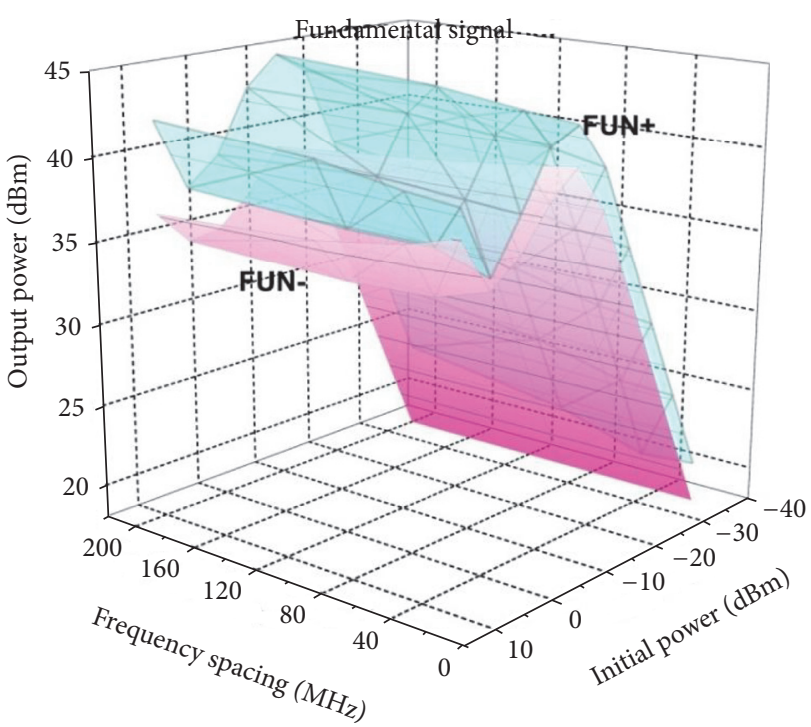

Figure 2: The 3D AM/AM curves of FUN- and FUN+ according to the increasing fundamental frequency spacing.

$(-20 \mathrm{dBm})$, besides, nonlinear phenomena emergence is delayed. So the fundamental frequency is set as $1.5 \mathrm{GHz}$ in the paper.

The FUN- in two-tone frequencies is set to $1.5 \mathrm{GHz}$; frequency spacing is increased from 0 to $200 \mathrm{MHz}$; the 3D AM/AM curve of the two-tone fundamental signal according to the increasing fundamental frequency spacing is shown in Figure 2. With the increase of fundamental frequency spacing, the output power of FUN+ presents a slightly increasing trend, but FUN- reduces obviously, and the 3D curves differentiation is more and more evident. When frequency spacing is set as $20 \mathrm{MHz}$, output powers of FUN- and FUN+ under saturation condition are 42.81 and $43.24 \mathrm{dBm}$. The 


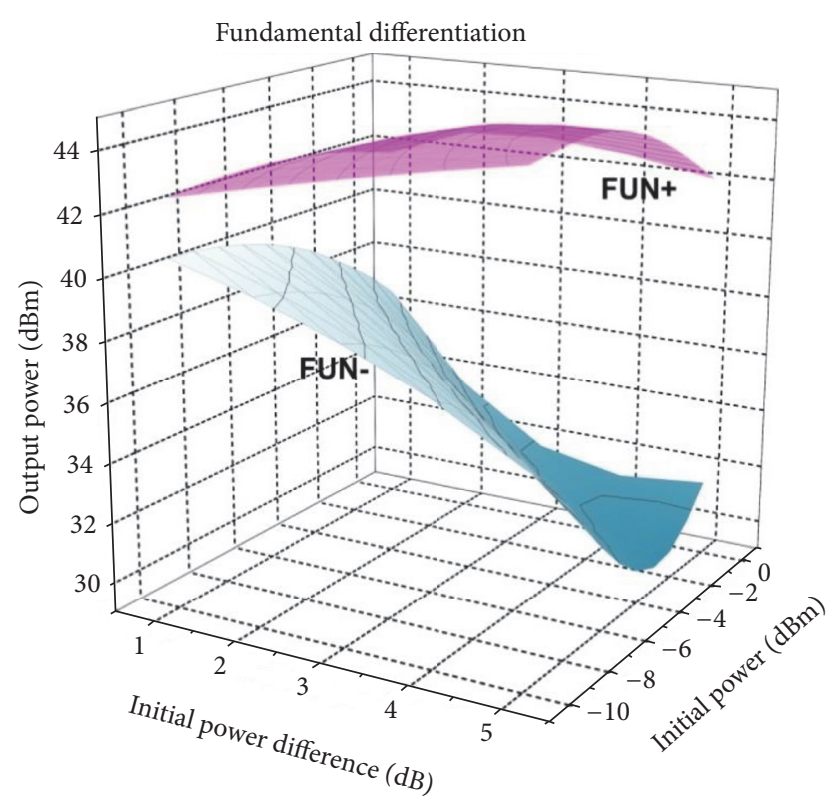

(a)

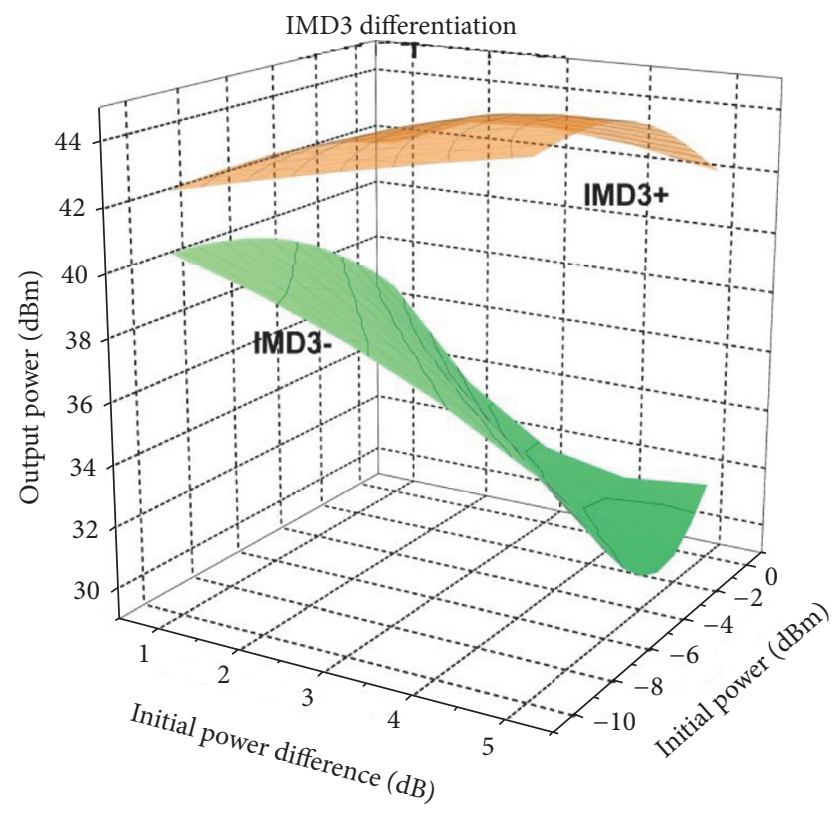

(b)

Figure 3: (a) 3D plot of fundamental differentiation; (b) 3D plot of IMD3 differentiation according to initial power and power difference.

difference between FUN- and FUN+ is the smallest, and amplification effect is more balanced; therefore fundamental frequency spacing is selected as $20 \mathrm{MHz}$.

It can be seen from Figure 3 that FUN- is severely suppressed and FUN+ remain stable according to the increase of relative power; the phenomenon of serious differentiation between them occurs. The difference of fundamental output power reaches the maximum at $14.23 \mathrm{~dB}$ when initial power is $-4 \mathrm{dBm}$ and power difference is $5 \mathrm{~dB}$. The trend of IMD3 change is similar to that of fundamental signal, IMD3+ rapidly increase, and IMD3- significantly decrease according to the increase of relative power. The fundamental energy is transferred to nonlinear distortion component after intermodulation, and nonlinear distortion phenomenon of STWT is more serious. So the initial power of two-tone fundamental is assumed to be equal, and relative power of two-tone fundamental signal is zero.

3.2. Simultaneous Suppression for the IMD3 and IMD5. On the basis of the previous research achievements on fundamental signal parameters in STWT, the initial twotone fundamental frequencies $\omega_{1}$ and $\omega_{2}$ are set as 1.50 and $1.52 \mathrm{GHz}$; phase and amplitude are $0.0^{\circ},-10.0 \mathrm{dBm}$. The IMD3 signal $\left(2 \omega_{2}-\omega_{1}, 1.54 \mathrm{GHz}\right)$ is introduced into the input port of STWT; the simulation experiment can precisely show the nonlinear characteristic of STWT according the output power responses to phase and amplitude scan of injected signal. Power scanning range is $-28 \sim-17 \mathrm{dBm}$ and phase scanning range is $270^{\circ} \sim 310^{\circ}$. It can be seen from Figure 4 that IMD3+ output power can be reduced $39.3 \mathrm{~dB}$ while amplitude and phase of injected IM3 signal are $-21 \mathrm{dBm}$ and $312^{\circ}$; then minimum output power of IMD3+ is $-9.6 \mathrm{dBm}$, but output power of IMD5+ is $17.9 \mathrm{dBm}$. While the amplitude

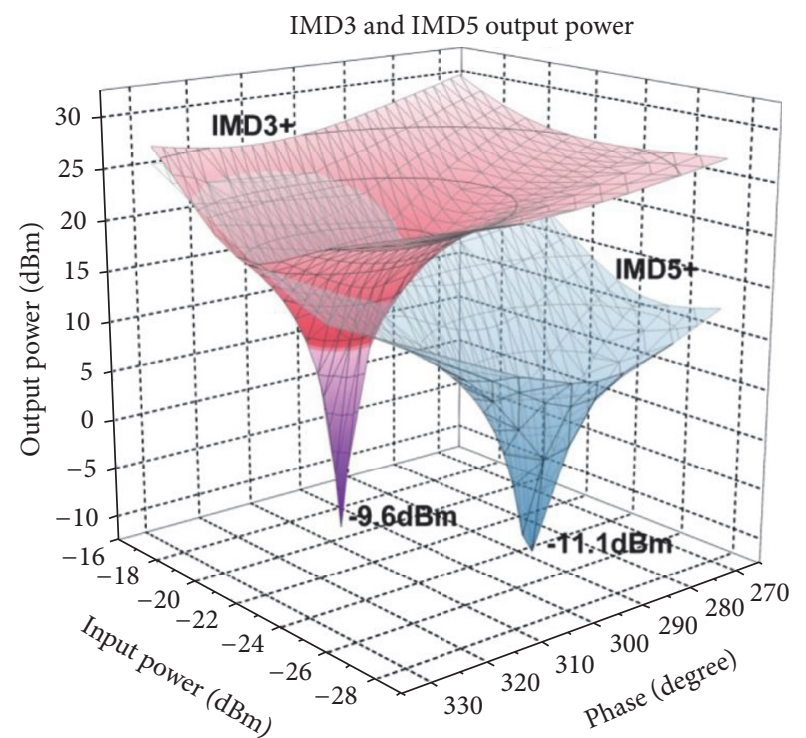

FIGURE 4: The 3D plot of the output power at IMD3 and IMD5 according to the phase and amplitude of injected IM3+ signal.

and phase of injected IMD3 signal be adjusted to $-25 \mathrm{dBm}$ and $292^{\circ}$, minimum output power of IMD5+ is $-11.1 \mathrm{dBm}$ and IMD5+ maximum suppression is $31.6 \mathrm{~dB}$; then output power of IMD3+ is $23.2 \mathrm{dBm}$. Figure 4 shows the 3D plots of the output power at IMD3 and IMD5 according to the phase and amplitude of injected IM3 signal. The initial output power of IMD5 is lower than that of IMD3 under the same injection condition; both gradually decrease and reach the only minimum when the injected signal parameters were adjusted to a certain range. The 3D plot presents a funnelshaped structure. There are significant differences among 


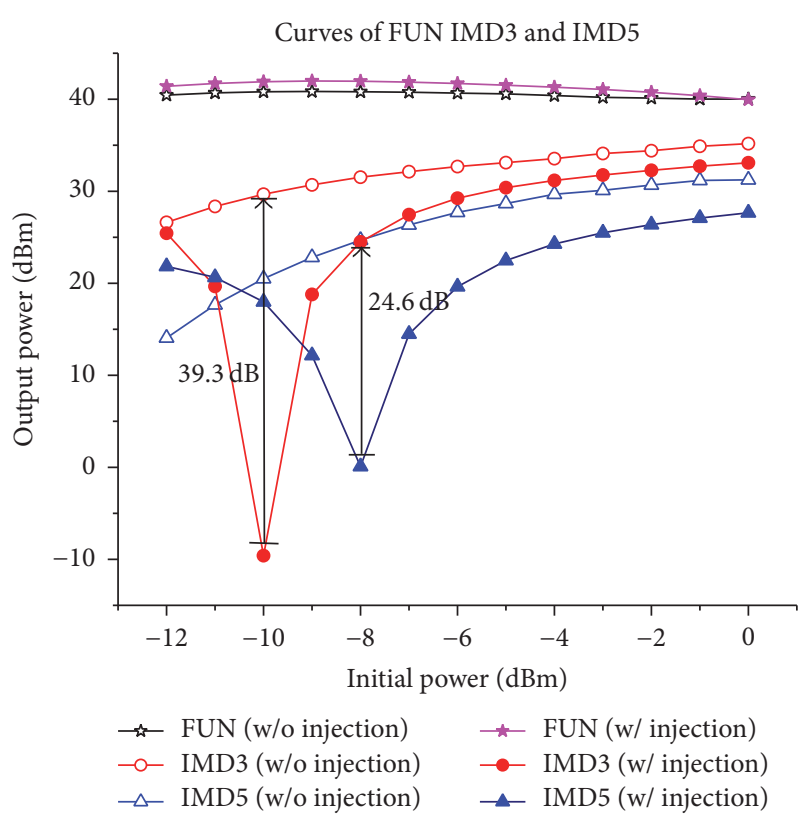

FIGURE 5: The output power of FUN, IMD3, and IMD5 according to initial power variation under optimum IM3 injection and without IM3 injection.

the injected parameters which make IMD3 and IMD5 reach minimum; the optimal signal injection conditions for IMD3 and IMD5 are not consistent.

The amplitude and phase of the injected IM3 signal are kept at $-21 \mathrm{dBm}, 312^{\circ}$, which produce maximum suppression for IMD3. Initial power of fundamental increases from -12 to $0 \mathrm{dBm}$ and phase is kept at $0^{\circ}$. The comparison of computation results of output powers at the fundamental, IMD3, and IMD5 under optimum IM3 injection and without IM3 injection is completed in this paper. It can be seen from Figure 5 that the fundamental output power remains constant without IM3 injection, and the curve of IMD5 and IMD3 increased slowly with the increase of initial power. The variation situation of output power at the fundamental, IMD3, and IMD5 under optimum IM3 injection and without IM3 injection can be observed by changing fundamental initial power, and computation results show that IMD3+ output power decreases rapidly when initial power increases from $-12 \mathrm{dBm}$, and maximum suppression $39.3 \mathrm{~dB}$ is obtained at initial power of $-10 \mathrm{dBm}$. The condition also causes the IMD5+ output power to slightly fall to $17.9 \mathrm{dBm}$ and does not achieve maximum suppression. When the initial power increases from $-10 \mathrm{dBm}$ to $-8 \mathrm{dBm}$, IMD5+ achieved maximum suppression of $24.6 \mathrm{~dB}$; in this process, IMD3+ output power increases from $-9.2 \mathrm{dBm}$ to $24.5 \mathrm{dBm}$. Therefore, the parametrical conditions of initial power for IMD3 and IMD5 maximum suppression are completely different under IMD3 signal injection.

The optimal signal injection conditions for IMD3 and IMD5 are not consistent. So we need to find the phase and amplitude of injected signal that ensure the minimum relative power of IMD3 to IMD5, so that the simultaneous suppression for the IMD3 and IMD5 is observed by signal injection.
3.3. Comparison on Simultaneous Suppression Effect by IM3 and $2 H D$ Injection. Based on simulation results, IMD3 has larger output power level compared to IMD5 and more close to fundamental frequency, so IMD3 suppression is a more important work for improving the performance of STWT. IMD3 to IMD5 ratio (TFR) represents relative output power of IMD3 to IMD5 and is set as the parameter to evaluate the performance of signal injection technique.

The initial two-tone fundamental frequencies are set as $1.50 \mathrm{GHz}$ and $1.52 \mathrm{GHz}$; amplitude and phase are $0.0^{\circ}$, $-10.0 \mathrm{dBm}$. The amplitude and phase of the injected IM3+ signal are kept at $-21 \mathrm{dBm}, 312^{\circ}$; the minimum is $-27.6 \mathrm{~dB}$ when the power of injected IM3+ increases from $-28 \mathrm{dBm}$ to $-17 \mathrm{dBm}$ and phase is kept from $270^{\circ}$ to $310^{\circ}$. This condition represents the IMD3 to IMD5 relative power at corresponding frequency point (higher or lower order) achieving the minimum and making the optimum simultaneous suppression for both IMD3 and IMD5 of STWT; then the IMD3+ and IMD5+ output power is $-9.6 \mathrm{dBm}$ and $17.9 \mathrm{dBm}$, respectively.

Second harmonic $\left(2 \omega_{2}\right)$ injection can also significantly suppress IMD3 and IMD5 according to the previous research results; then whether the effect of $2 \mathrm{HD}$ injection is any better on simultaneous suppression in both IMD3 and IMD5 compared with IM3 injection is an important research topic. The power of injected $2 \mathrm{HD}$ increases from 10 to $30 \mathrm{dBm}$, phase scanning range is slightly expanded to $270^{\circ} \sim 330^{\circ}$, minimum value of $\mathrm{TFR}_{\mathrm{IM} 3 \text { to IM5 }}$ is $-20.19 \mathrm{~dB}$, and then the output power of IMD3+ and IMD5+ is $-4.6 \mathrm{dBm}$ and $15.57 \mathrm{dBm}$, respectively. The amplifier performance of STWT by $2 \mathrm{HD}$ injection compared to IM3 injection has also been weakened slightly.

Minimum of $\mathrm{TFR}_{\mathrm{IM} 3 \text { to IM5 }}(-27.6 \mathrm{~dB})$ by IM3 injection is lower than that $(-20.19 \mathrm{~dB})$ of $2 \mathrm{HD}$ injection, and the total power of the nonlinear distortion component is lower; its optimizing effect on simultaneous suppression of IMD3 and IMD5 is quite obvious. Figures 6 and 7 show that the initial power of the injected IM3 and 2HD is very different; the 3D curve of TFR changes smoothly with variation of input power but changes drastically with variation of input phase. According to the known research results, input phase plays more important role in the function of intermodulation in TWT compared to input power; it has more impact on the simultaneous suppressions in both IMD3 and IMD5. The phase range which makes TFR gradually go down to the minimum is all the $270^{\circ} \sim 330^{\circ}$ whether $2 \mathrm{HD}$ or IM3 injection, so the phase range can make the relative power of IMD3 and IMD5 achieve minimum value.

Fundamental frequency spacing for simultaneous suppression in both IMD3 and IMD5 of STWT is studied through the simulation method under the optimal signal injection for $\mathrm{TFR}_{\mathrm{IM} 3 \text { to IM5 }}$. We measure the IMD3 and IMD5 with and without injection for fundamental frequency spacing as shown in Figure 8. The center frequency of RF signals is $1.5 \mathrm{GHz}$, and then channel spacing is changed from $1 \mathrm{MHz}$ to $10 \mathrm{MHz}, 20 \mathrm{MHz}, 40 \mathrm{MHz}, 60 \mathrm{MHz}, 80 \mathrm{MHz}$, and $100 \mathrm{MHz}$, and the initial two-tone fundamental power is set as $-10 \mathrm{dBm}$. The suppression of IMD3+ was close to $40 \mathrm{~dB}$ under fundamental frequency spacing of $20 \mathrm{MHz}$ after optimal signal injection in Figure 8. With the increase of 


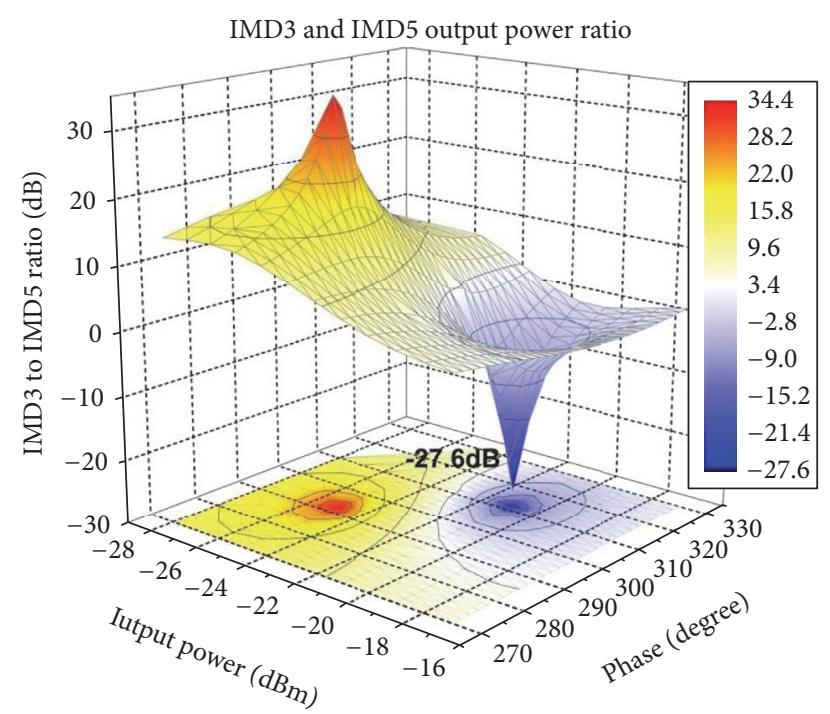

FIgURE 6: The 3D plot of $\mathrm{TFR}_{\mathrm{IM} 3 \text { to IM5 }}$ according to the phase and amplitude of injected IM3+ signal.

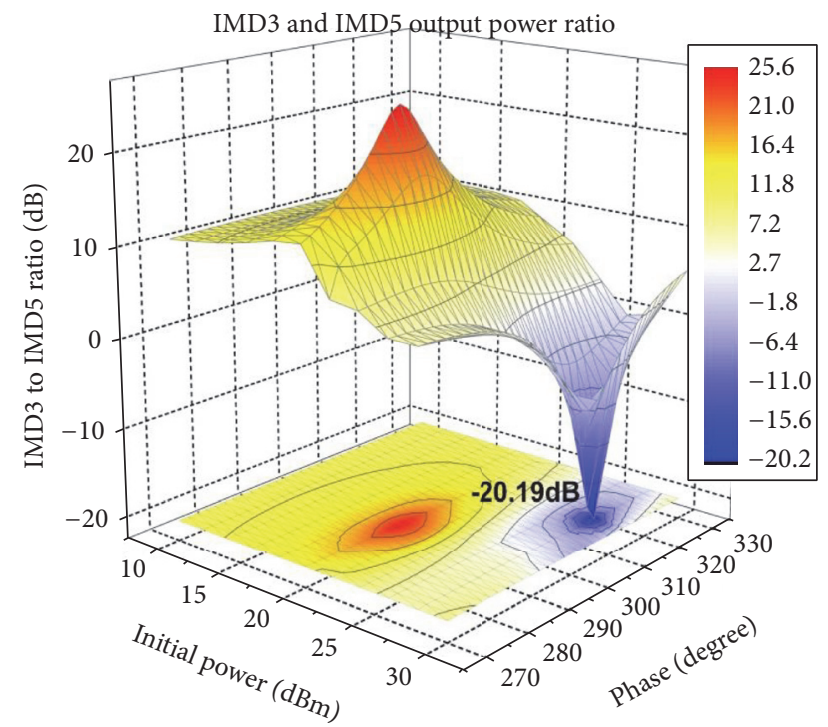

FIgure 7: The $3 \mathrm{D}$ plot of $\mathrm{TFR}_{\mathrm{IM} 3}$ to IM5 according to the phase and amplitude of injected 2HD.

fundamental frequency spacing, the suppression effect of IMD3+ is weakened, but the suppression of $28 \mathrm{~dB}$ can still be obtained even at the $100 \mathrm{MHz}$. Meanwhile, IMD5 can also be suppressed at $1.85 \sim 5.35 \mathrm{~dB}$; fundamental output power is almost constant. Therefore, IMD3 and IMD5 of STWT can be significantly suppressed in range of $0 \sim 100 \mathrm{MHz}$ of fundamental frequency spacing under the optimal signal injection for $\mathrm{TFR}_{\mathrm{IM} 3 \text { to IM5 }}$.

\section{Experiment and Analysis}

The vector network analyzer (VNA) can precisely describe the property of a device according to its amplitude/phase

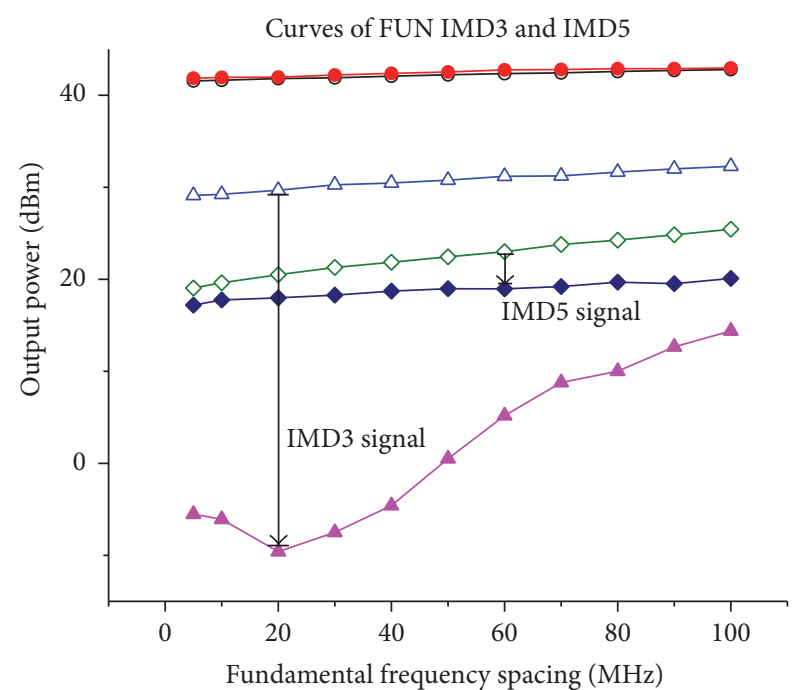

$\multimap$ FUN (Without Injection) $\multimap$ IMD3 (Optimum Injection)
$\rightarrow$ FUN (Optimum Injection) $\multimap$ IMD5 (Without Injection)
$\rightarrow$ IMD3 (Without Injection) $\rightarrow$ IMD5 (Optimum Injection)

FIGURE 8: Suppression situation for IMD3+ and IMD5+ under optimal signal injection according to fundamental frequency spacing $(1 \mathrm{MHz} 100 \mathrm{MHz})$.

responses to frequency and power scan of measurement signals.

The rated output power of the STWT which is used in the experiment is $120 \mathrm{~W}(55 \mathrm{dBm})$, its gain is $50 \mathrm{~dB}$, and working frequency band is $1.4-1.6 \mathrm{GHz}$. The experiment block diagram of simultaneous suppressions in both IMD3 and IMD5 is shown in Figure 9. The initial two-tone fundamental frequencies are set as 1.50 and $1.52 \mathrm{GHz}$, and lower $2 \mathrm{HD}$ $(3.00 \mathrm{GHz})$ and IM3 $(1.48 \mathrm{GHz})$ are injected into the input port of STWT. The power and phase are adjusted by signal generator and phase shifter, the nonlinear component of injected signal and fundamental cancel one another in the output when they are equal in output power and opposite in phase. The output power of STWT is 45.9 and $46.0 \mathrm{dBm}$, and the power of IMD3- and IMD3+ is 33.9 and $33.2 \mathrm{dBm}$, respectively, under no signal injection.

4.1. IMD3 Injection. The IMD3- $(1.48 \mathrm{GHz})$ is introduced into the input port of STWT, and phase and amplitude are adjusted by signal generator and phase shifter. When the injected IM3- power is $-5.86 \mathrm{dBm}$, the minimum value of $\mathrm{TFR}_{\mathrm{IM} 3 \text { to IM5 }}$ is obtained at $2.1 \mathrm{~dB}$. Output signal spectrum under optimal signal injection for IMD3 and IMD5 of STWT is shown in Figure 10. Then the components of the output signal are $46.7 \mathrm{dBm}$ (FUN-), $8.8 \mathrm{dBm}$ (IMD3-), and $6.7 \mathrm{dBm}$ (IMD5-).

Maximum suppression of $25.1 \mathrm{~dB}$ for IMD3- can be observed by vector network analyzer; then the suppression for IMD5- is $17.3 \mathrm{~dB}$. The total output power of STWT is $121.4 \mathrm{~W}$, and it also has good characteristics of gain. The experiment proves that IMD3 signal injection can make 


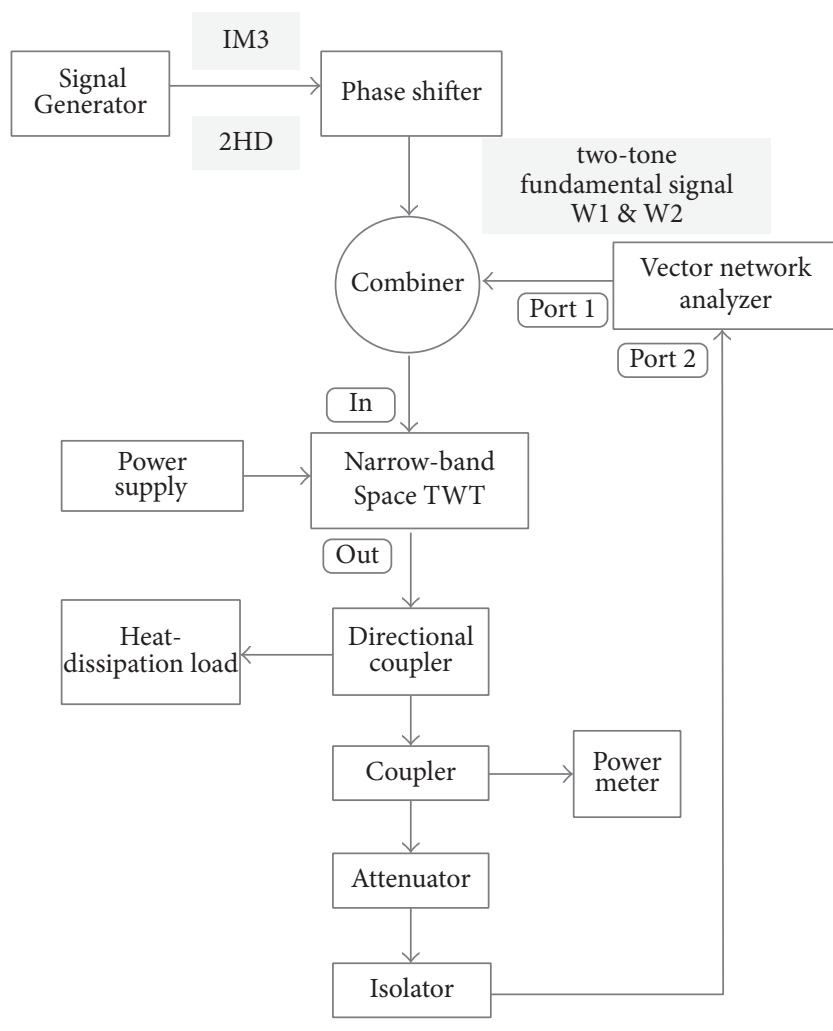

FIGURE 9: The experiment block diagram of simultaneous suppressions in both IMD3 and IMD5.

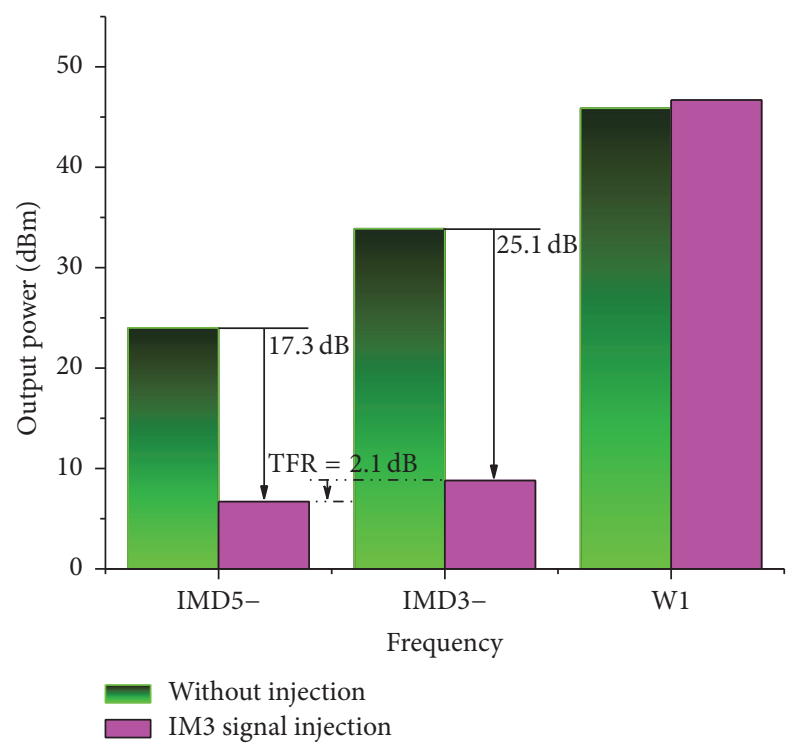

FIgURE 10: The output signal spectrum under optimal IMD3injection of simultaneous suppression for IMD3 and IMD5.

simultaneous suppression for the IMD3 and IMD5 at corresponding frequency of STWT.

4.2. $2 \mathrm{HD}$ Injection. Alternatively, $2 \mathrm{HD}(3.0 \mathrm{GHz})$ is introduced into the STWT; when the amplitudes are adjusted to $13 \mathrm{dBm}$, the minimum value of TFR $\mathrm{IM} 3$ to IM5 $_{5}$ is $12.5 \mathrm{~dB}$.

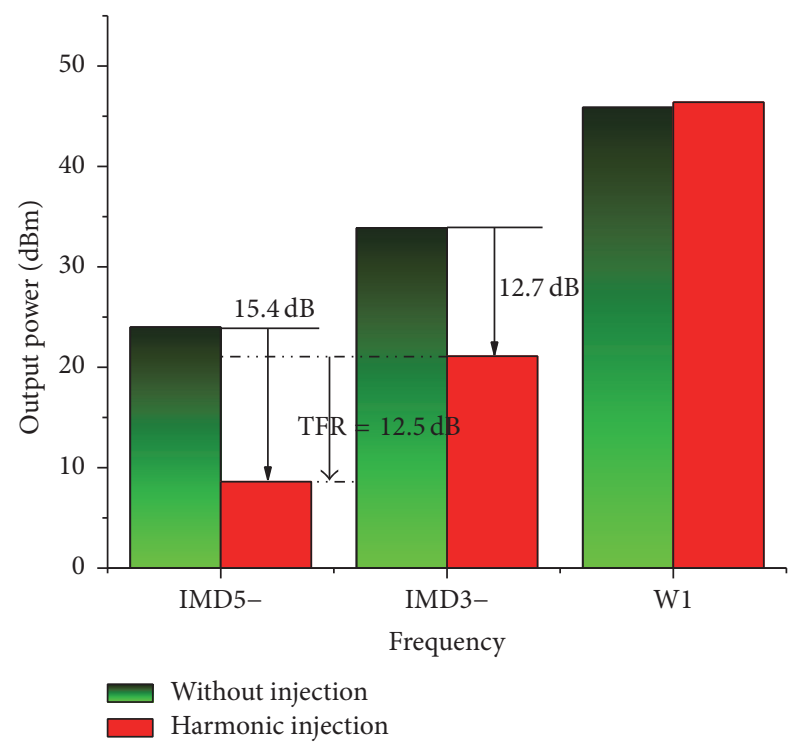

FIGURE 11: The output signal spectrum under optimal 2HD injection of simultaneous suppression for IMD3 and IMD5.

TABLE 1: The output power, suppression, and corresponding TFR under optimum IM3 injection and 2HD injection in simulation.

\begin{tabular}{lcccccc}
\hline Mode & $\begin{array}{c}\text { TFR } \\
(\mathrm{dB})\end{array}$ & \multicolumn{2}{c}{$\begin{array}{c}\text { Output power } \\
(\mathrm{dBm})\end{array}$} & \multicolumn{3}{c}{$\begin{array}{c}\text { Suppression } \\
(\mathrm{dB})\end{array}$} \\
& & IMD5 & IMD3 & FUN & IMD5 & IMD3 \\
\hline IM3 & -27.6 & 17.9 & -9.6 & 43.3 & 2.6 & 39.3 \\
2HD & -20.2 & 15.6 & -4.6 & 42.9 & 4.9 & 34.3 \\
\hline
\end{tabular}

TABLE 2: The output power and corresponding TFR under IM3 injection and $2 \mathrm{HD}$ injection in experiment.

\begin{tabular}{lcccccc}
\hline Mode & $\begin{array}{c}\text { TFR } \\
(\mathrm{dB})\end{array}$ & \multicolumn{2}{c}{$\begin{array}{c}\text { Output power } \\
(\mathrm{dBm})\end{array}$} & \multicolumn{3}{c}{$\begin{array}{c}\text { Suppression } \\
(\mathrm{dB})\end{array}$} \\
& & IMD5 & IMD3 & FUN & IMD5 & IMD3 \\
\hline IM3 & 2.1 & 6.7 & 8.8 & 46.7 & 17.3 & 25.1 \\
2HD & 12.5 & 8.6 & 21.1 & 46.4 & 15.4 & 12.7 \\
\hline
\end{tabular}

The output signal spectrum under optimal signal injection for IMD3 and IMD5 of STWT is shown in Figure 11. Then the components of the output signal are $46.7 \mathrm{dBm}$ (FUN-), $8.8 \mathrm{dBm}$ (IMD3-), and $6.7 \mathrm{dBm}$ (IMD5-). Maximum suppression of $12.7 \mathrm{~dB}$ for IMD3- can be observed. Besides, suppression for IMD5- is $15.4 \mathrm{~dB}$, and the total output power also is improved by $0.5 \mathrm{~dB}$. The results of experiments suggest that $2 \mathrm{HD}$ injection can make simultaneous suppression for the IMD3 and IMD5 at corresponding frequency of STWT. The output power and corresponding TFR values under optimum IM3 and 2HD signal injection by means of simulation and experiment are shown in Tables 1 and 2 , respectively.

4.3. Comparison between Simulation and Experiment. The value of TFR and IMD suppression achieved experimentally is limited by resolution bandwidths of signal generator 
TABLE 3: Comparison of TFR and IMD suppression between simulation and experiment.

\begin{tabular}{|c|c|c|c|c|c|c|}
\hline \multirow{3}{*}{ Mode } & \multicolumn{3}{|c|}{ Simulation } & \multicolumn{3}{|c|}{ Experiment } \\
\hline & \multirow{2}{*}{$\begin{array}{l}\text { TFR } \\
(\mathrm{dB})\end{array}$} & \multicolumn{2}{|c|}{$\begin{array}{l}\text { Suppression } \\
\text { (dB) }\end{array}$} & \multirow{2}{*}{$\begin{array}{l}\text { TFR } \\
(\mathrm{dB})\end{array}$} & \multicolumn{2}{|c|}{$\begin{array}{l}\text { Suppression } \\
(\mathrm{dB})\end{array}$} \\
\hline & & IMD5 & IMD3 & & IMD5 & IMD3 \\
\hline IM3 & -27.6 & 2.6 & 39.3 & 2.1 & 17.3 & 25.1 \\
\hline $2 \mathrm{HD}$ & -20.2 & 4.9 & 34.3 & 12.5 & 15.4 & 12.7 \\
\hline
\end{tabular}

and phase shifter; in particular, the suppression of IMD is particularly sensitive to the phase adjustment of the phase shifter, and the amplitude and phase of injected signal can be tuned precisely to get simultaneous suppression.

Comparison of TFR and IMD suppression between simulation and experiment is shown in Tables 1 and 3. The TFR is 2.1 and $12.5 \mathrm{~dB}$ under IM3 and $2 \mathrm{HD}$ injection observed experimentally, much lower than the simulation results of TFR (-27.6 and -20.2). Specifically, when the minimum value of TFR is $2.1 \mathrm{~dB}$ under IM3 injection, suppression of $25.1 \mathrm{~dB}$ for IMD3 can be observed in experiment, but suppression for IMD3 is $39.3 \mathrm{~dB}$ under the same simulation condition (minimum TFR is $-27.6 \mathrm{~dB}$ ). The precision of input amplitude and phase was limited by signal generator and phase shifter to match the amount of suppression observed by means of simulation. In spite of these factors, the experiment and simulation are in very good qualitative agreement, the tendency that TFR with IM3 injection is lower than that with $2 \mathrm{HD}$ injection for STWT is very clear, and the results that the simultaneous suppression effect by IM3 injection is better than $2 \mathrm{HD}$ injection in simulation are in good agreement with the experimental data.

\section{Conclusion}

This paper introduces the definition of IMD3 to the IMD5 ratio (TFR), by choosing the optimal operating point of the injected amplitude and phase where the TFR has minimum variations, and derives the signal injection conditions for simultaneous suppression in the IMD3 and IMD5. According to the research on theoretical analysis and computer simulation, the optimal amplitude and phase parameters of the injected 2HD and IMD3 for maximum simultaneous suppressions are obtained. Then an experiment system is established based on vector network analyzer; the optimal TFR are $2.1 \mathrm{~dB}$ and $12.5 \mathrm{~dB}$, respectively, by $2 \mathrm{HD}$ and IM3 injection. The output power of IMD3 and IMD5 was decreased. TFR with IM3 injection is smaller than that with $2 \mathrm{HD}$ injection in STWT. The experiments system is more simple and easy to operate. Thus, the IM3 injection performs better than $2 \mathrm{HD}$ injection to suppress IMD5s for narrowband STWT.

\section{Disclosure}

The corresponding author is Professor Kewen Xia.

\section{Competing Interests}

The authors declare that there is no conflict of interests regarding the publication of this paper.

\section{Acknowledgments}

This work was supported by Hebei Province Natural Science Foundation (no. E2016202341), Hebei Province Foundation for Returned Scholars (no. C2012003038), and Research Project of Science and Technology for Hebei Province Higher Education Institutions (no. BJ20 1401).

\section{References}

[1] J. G. Wöhlbier, J. H. Booske, and I. Dobson, "The multifrequency spectral Eulerian (MUSE) model of a traveling wave tube," IEEE Transactions on Plasma Science, vol. 30, no. 3, pp. 1063-1075, 2002.

[2] J. G. Wohlbier, Nonlinear distortion and suppression in traveling wave tubes Insights and methods [Ph.D. thesis], University of Wisconsin, Madison, Wis, USA, 2003.

[3] J. G. Wöhlbier, J. H. Booske, and I. Dobson, "On the physics of harmonic injection in a traveling wave tube," IEEE Transactions on Plasma Science, vol. 32, no. 3 I, pp. 1073-1085, 2004.

[4] J. Plouin, J.-M. Rax, F. Andre, and Y. Thaler, "Improvement of the output power in TWTs by harmonic injection," IEEE Transactions on Electron Devices, vol. 52, no. 5, pp. 783-789, 2005.

[5] M. Wirth, A. Singh, J. Scharer, and J. Booske, "Third-order intermodulation reduction by harmonic injection in a TWT amplifier," IEEE Transactions on Electron Devices, vol. 49, no. 6, pp. 1082-1084, 2002.

[6] A. Singh, J. E. Scharer, J. H. Booske, and J. G. Wöhlbier, "Secondand third-order signal predistortion for nonlinear distortion suppression in a TWT," IEEE Transactions on Electron Devices, vol. 52, no. 5, pp. 709-717, 2005.

[7] A. Singh, Experimental investigation of TWT nonlinearities and distortion suppression by signal injection [M.S. thesis], University of Wisconsin-Madison, Madison, Wis, USA, 2003.

[8] T.-K. Lee, Y.-T. Moon, H.-S. Kim, and Y.-W. Choi, “Theoretical analysis and realization of optoelectrical predistortion optical transmitter for the simultaneous suppression of IM3 and IM5 signal," Optics Communications, vol. 285, no. 10-11, pp. 26972701, 2012.

[9] S. Piramasubramanian and M. Ganesh Madhan, "Simultaneous reduction of IMD3 and IMD5 in bisection laser diode by feedback second harmonic injection," Optics Communications, vol. 328, pp. 151-160, 2014.

[10] S. Piramasubramanian, Novel distortion reduction and pulse generation schemes in gain lever laser diode [Ph.D. thesis], Anna University, Chennai, India, 2014.

[11] S. Piramasubramanian and M. Ganesh Madhan, "A novel distortion reduction scheme for multiple quantum well gain lever laser diodes," Journal of Optics, vol. 15, no. 5, Article ID 055501, pp. 527-535, 2013.

[12] X. F. Liang, S. L. Cai, and M. G. Huang, "Analysis of harmonic injection on the bandwidth of TWTs," Vacuum Electronics, vol. 3, pp. 23-26, 2008.

[13] A. Bakry and M. Ahmed, "Harmonic and intermodulation distortions and noise associated with two-tone modulation of 
high-speed semiconductor lasers," Physics of Wave Phenomena, vol. 24, no. 1, pp. 64-72, 2016.

[14] X. Ding and J. Li, "Second harmonic injection for restraining third-order intermodulation in traveling wave tube," High Power Laser and Particle Beams, vol. 21, no. 11, pp. 1691-1695, 2009.

[15] Y. Xu, Predistortion technology of multi-band broadband wireless communication system [M.S. thesis], Xiamen University, 2013.

[16] X. Zhang, K. Tang, S. Li, K. Xia, and D. Zhao, "Design of slow-wave structure based on multi-objective quantum particle swarm optimization algorithm with inertia weight," Chinese Journal of Vacuum Science \& Technology, vol. 30, no. 6, pp. 651656, 2010

[17] D. Zhao, K. Tang, and S. Li, "Third-order intermodulation distortion suppression based on signal injection in space TWT," Vacuum Electronics, vol. 3, pp. 4-10, 2010.

[18] N.-P. Liu, D.-M. Zhao, and K.-W. Xia, "Research on fifth-order intermodulation suppression by signal injection in STWT," Vacuum Electronics, no. 3, pp. 1-5, 2011.

[19] C. S. Aitchison, M. Mbabele, and M. Reza Moazzam, "Improvement of third-order intermodulation product of RF and microwave amplifiers by injection," IEEE Transactions on Microwave Theory and Techniques, vol. 49, no. 6, pp. 1148-1154, 2001. 


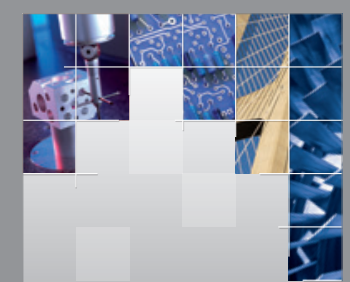

\section{Enfincering}
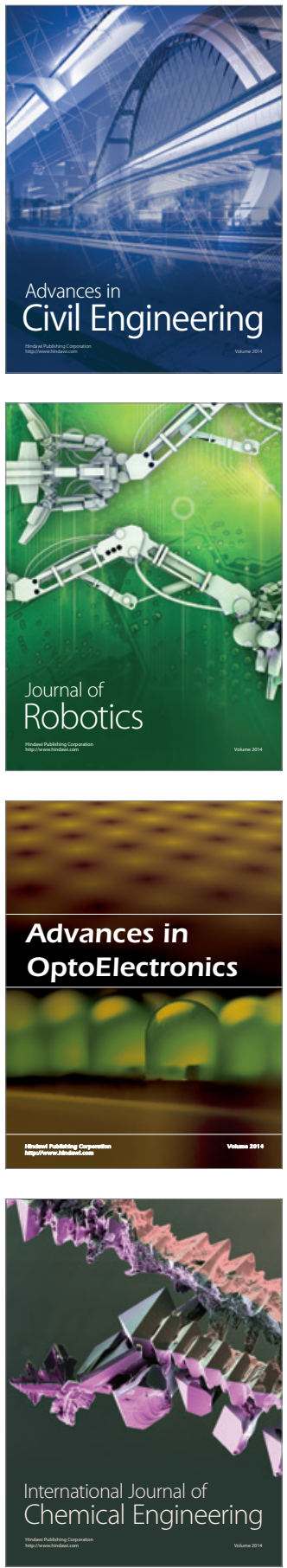

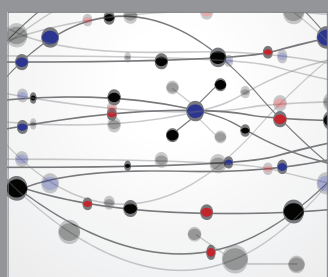

The Scientific World Journal

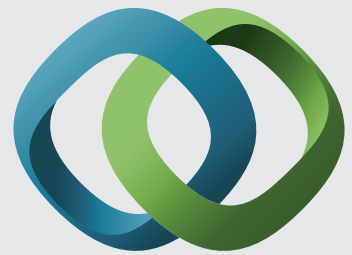

\section{Hindawi}

Submit your manuscripts at

https://www.hindawi.com
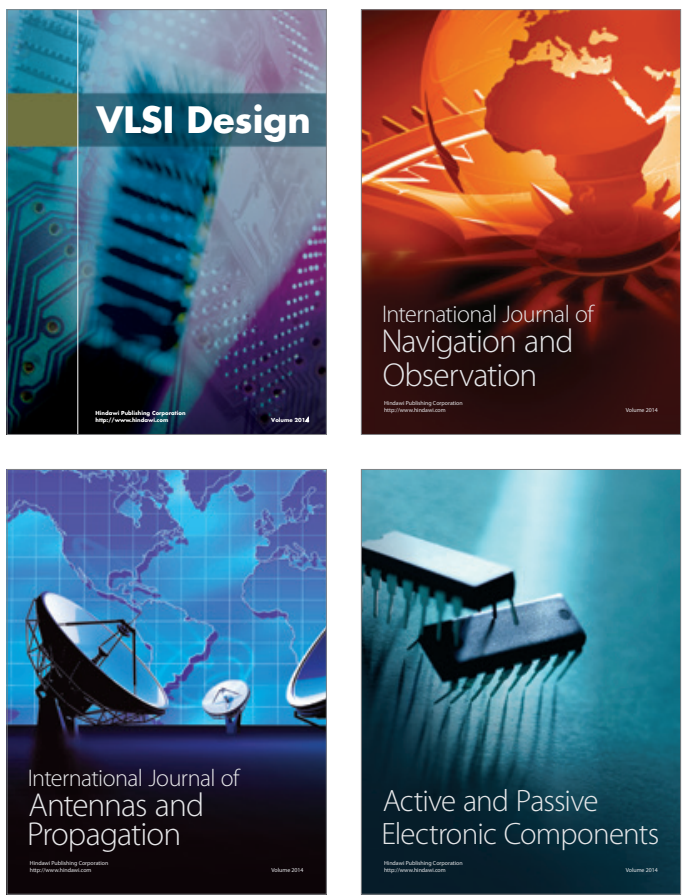
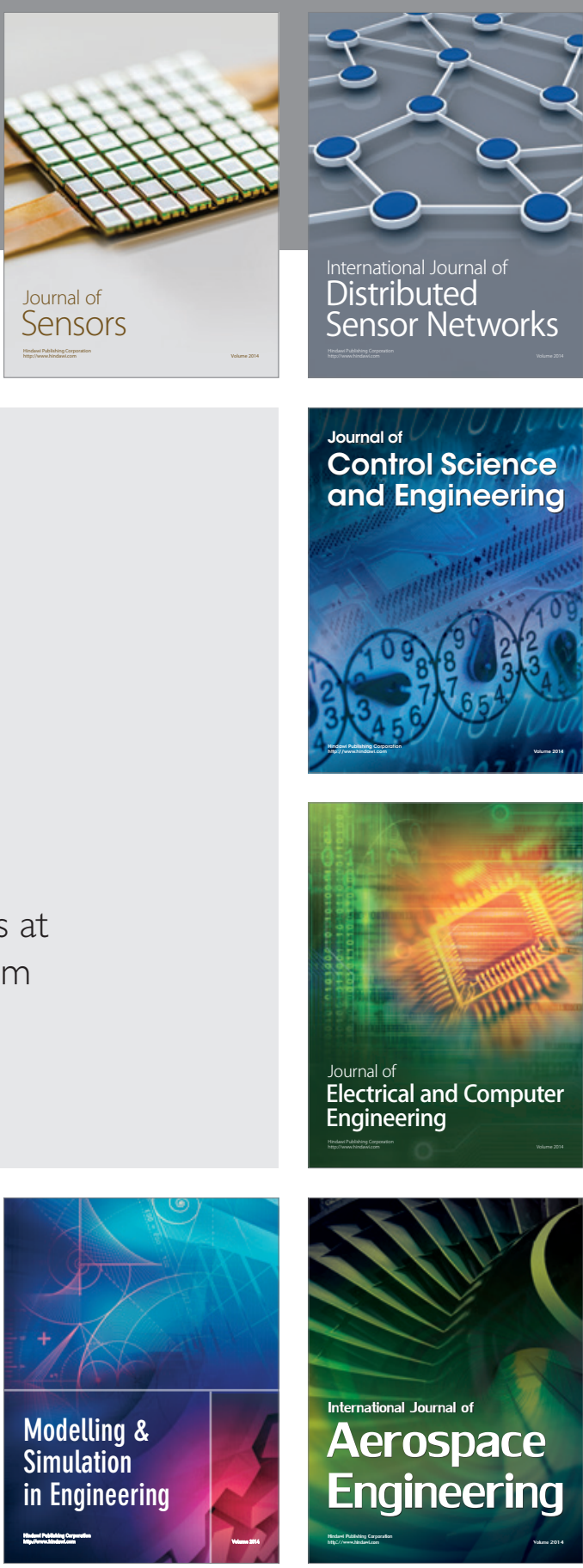

International Journal of

Distributed

Sensor Networks

$-$

Joumal of

Control Science

and Engineering
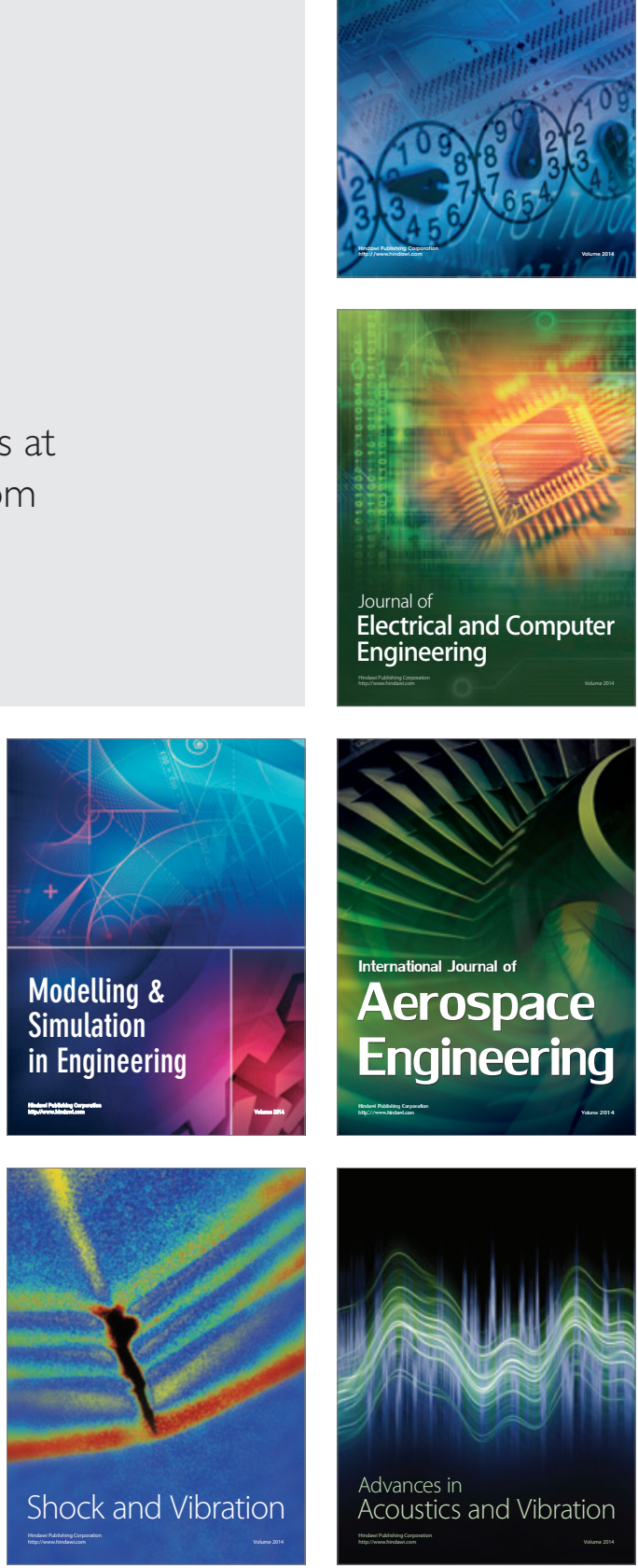